\title{
Path integral method for stochastic equations of financial engineering
}

\author{
Yanishevskyi V. S., Baranovska S. P. \\ Lviv Polytechnic National University, \\ 12 S. Bandera Str., 79013, Lviv, Ukraine
}

(Received 13 August 2021; Accepted 19 January 2022)

\begin{abstract}
The integral path method was applied to determine certain stochastic variables which occur in problems of financial engineering. A stochastic variable was defined by a stochastic equation where drift and volatility are functions of a stochastic variable. As a result, for transition probability density, a path integral was built by substituting variables Wiener's path integral (Wiener's measure). For the stochastic equation, Ito rule was applied in order to interpret a stochastic integral. The path integral for transition probability density was also found as a result of the Fokker-Planck equation solution, corresponding to the stochastic equation. It was shown that these two approaches give equivalent results.
\end{abstract}

Keywords: stochastic equation, Fokker-Plank equation, transition probability density, path integrals.

2010 MSC: 91B70, 91G30, 81S40

DOI: $10.23939 / \mathrm{mmc} 2022.01 .166$

\section{Introduction}

Stochastic processes are at the foundation of many phenomena: physical, economic, financial, social, etc. $[1,2]$. To describe them, one uses stochastic differential equations or an associated with them Fokker-Plank equations. The stochastic differential equation describes the dynamics of a certain value, and the Fokker-Plank equation determines the transition probability density of stochastic variable distribution. Stochastic equations are based on Brownian motion, which was used to describe stock pricing dynamics and Brownian particle movement. The mathematic theory behind the Brownian motion was founded by Wiener. In order to find average values of stochastic process characteristics, one should integrate over Wiener measure, which is defined for space of all trajectories of stochastic variable dynamics [3]. Integral over Wiener's measure is a path integral of the simplest stochastic equation, which describes Brownian motion or Wiener's process.

Apart from that, path integral (integral over trajectories) is used in solving problems of quantum mechanics and is based on Feynman-Kac theorem for Schrödinger equation solution [3-5]. The same form makes sense for the Fokker-Plank equation solution. It is understood that path integrals that are introduced for stochastic equations based on Wiener's measure or for representation of solutions of Fokker-Plank the equation should be equivalent and give the same description results.

Application of path integral to financial problems was considered in [6-8], where the construction of path integral over Wiener's measure was pointed out. As it turned out, for stochastic equations with constant volatility (7) (in physical research of Langevin equations with adaptive white noise [1]) such construction is achieved unambiguously. However, for stochastic equations with volatility which is a function of stochastic variable (1) (or Langevin equations with multiplicative noise), the construction of path integral is ambiguous [7]. Under such conditions, the rules of Ito, Stratonovich or more generic schemas of stochastic integral determination are considered. The form of the Fokker-Plank equation depends on the choice of the scheme of stochastic integral determination. As a result, path integrals that depend on the choice of stochastic integral rule occur [7].

Despite a vast quantity of works dedicated to the theme, there are a number of inconsistencies and contradictions for received results [9-12]. In particular, in many physical applications during construction of path integral for Fokker-Plank equation, the analogies that include some arbitrariness are 
used [9-11]. In works [13,14] general schemes of discretization of Langevin equation with multiplicative noise are considered, the functional representations of transition probability density are obtained, and Onsager-Machlup functions are shown. However, the corresponding Fokker-Plank equations are not given. In work [15], a construction of forming functional for a finding of mean values that are described by the stochastic equation of (1) type was considered. However, no function which is handy in the application was given.

In this paper, a functional integral for the transition probability density of stochastic equation (1) was built. In order to determine stochastic integral, an approach for Ito scheme [7], which is usually applied to financial problems, was used. Unlike work [7] we built a functional integral of variables of equation (1). In addition, we introduced a solution to the Fokker-Plank equation for transition probability density in the form of functional integral and showed the equivalence of these two approaches.

\section{Construction of path integral for stochastic equation}

In problems of financial engineering, the price of stocks, assets, interest rate, and other attributes reveal the stochastic nature [16]. Dynamics of noted attributes is modeled with the help of stochastic equation which generally can be written as

$$
d r(\tau)=A(r(\tau)) d \tau+B(r(\tau)) d w(\tau)
$$

where $d w$ denotes a Wiener process. The stochastic variable $d w$ has a normal distribution with parameters $\langle d w\rangle=0,\left\langle d w^{2}\right\rangle=d t$. Values $A(r), B(r)$ define the drift and process volatility respectively and usually depend on the stochastic variable as well. Mostly the need of calculation of mean values over all realizations of stochastic process occurs, which is performed by integrating over the Wiener measure [3]

$$
\langle F(r(\tau))\rangle_{w}=\int_{w_{0}} d \mu(w) F(r(\tau))
$$

where $F(r(\tau))$ is a functional, which depends on variables $r(\tau)$ at moments of time $\tau \in\left[t_{0}, t\right]$. An element of Wiener's measure is defined by the following formula

$$
d \mu(w)=\mathcal{D} w(\tau) \exp \left(-\frac{1}{2} \int_{t_{0}}^{t}\left(\frac{w(\tau)}{d \tau}\right)^{2} d \tau\right), \quad \mathcal{D} w(\tau)=\prod_{\tau} \frac{d w(\tau)}{\sqrt{2 \pi d \tau}} .
$$

Direct computation in (2) consists of solution of stochastic equation (1) for $r(\tau)$ and it's substitution into (2) [17]. It is understood that the solution can be found only for the simple cases. That is why a more practical approach is in variable substitution in integral over Wiener measure, taking into account a connection between variables defined by equation (1)

$$
\int_{w_{0}} d \mu(w) F(r(\tau))=\int_{r_{0}} d \mu(r) F(r(\tau)) .
$$

Here $d \mu(r)$ denotes element of the measure received as a result of variable substitution. If functional $F(r(\tau))$ depends only on the value of variable $r(t)$ at boundary, then averaging in (2) reduces to integral with transition probability density $K\left(r, r_{0}, t, t_{0}\right)$

$$
\int_{r_{0}} d \mu(r) F(r(\tau))=\int d r F(r) K\left(r, r_{0}, t, t_{0}\right),
$$

where transition probability density determines through conditional measure

$$
K\left(r, r_{0}, t, t_{0}\right)=\int_{r_{0}}^{r} d \mu(r) .
$$


In problems of modeling of interest rate [16] the following integrals occur

$$
\int_{r_{0}} d \mu(r) F\left(\int_{t_{0}}^{t} r(\tau) d \tau\right)=\int d r \int_{r_{0}}^{r} d \mu(r) F\left(\int_{t_{0}}^{t} r(\tau) d \tau\right)
$$

Let's consider the problem of construction of path integral for the transition probability density of stochastic variable $r(\tau)$ based on (5) relation It is obvious that such transformations take place in (6) case.

Mentioned above transformations are unambiguous in case of constant volatility $B(r)=\sigma$

$$
d r(\tau)=A(r(\tau)) d \tau+\sigma d W(\tau)
$$

Construction of path integral for stochastic equation (7) is given in Appendix A.

As in the case of (7) equation let us write equation (1) in integral form

$$
w(\tau)-w\left(t_{0}\right)=\int_{t_{0}}^{\tau} \frac{d r\left(\tau^{\prime}\right)}{B\left(r\left(\tau^{\prime}\right)\right)}-\int_{t_{0}}^{\tau} \frac{A\left(r\left(\tau^{\prime}\right)\right)}{B\left(r\left(\tau^{\prime}\right)\right)} d \tau^{\prime} .
$$

First integral in left part of (8) is a stochastic integral $[2,7]$ which value depends on the approach used in computation of integral sum. For stochastic integrals rules corresponding to the different choices of points for integrated function in integral sum were proposed. Let's denote stochastic variable related to stochastic integral by $x(\tau)$

$$
x(\tau)-x\left(t_{0}\right)=\int_{t_{0}}^{\tau} \frac{d r}{B\left(r\left(\tau^{\prime}\right)\right)}=\varphi(r(\tau))-\varphi\left(r\left(t_{0}\right)\right) .
$$

Using Ito's formula we find a differential equation which is satisfied by value $x(\tau)$

$$
d x(\tau)=\varphi^{\prime}(r(\tau)) d r(\tau)+\frac{1}{2} \varphi^{\prime \prime}(r(\tau)) d r(\tau)^{2}=\left(\frac{A(r(\tau))}{B(r(\tau))}-\frac{1}{2} B^{\prime}(r(\tau))\right) d \tau+d w(\tau) .
$$

Formula (10) takes into account that $d r(\tau)^{2} \approx B(r(\tau))^{2} d \tau$. As it is seen, a stochastic differential equation with constant volatility corresponds to the stochastic variable $x(\tau)$. Such an approach of transitioning to the stochastic variable for which volatility is constant was proposed in [7]. In this case, representation of transition probability density by path integral is defined by formula (32). Let's rewrite equation (10) by substituting stochastic variable $r(\tau)$ with $x(\tau)$

$$
d x(\tau)=A_{\mathrm{ef}}(x(\tau)) d \tau+d w(\tau)
$$

with the following notation

$$
A_{\mathrm{ef}}(x(\tau))=\frac{A\left(\varphi^{-1}(x(\tau))\right)}{B\left(\varphi^{-1}(x(\tau))\right)}-\frac{1}{2} B^{\prime}\left(\varphi^{-1}(x(\tau))\right) .
$$

As a result for stochastic equation (11) it is received the next path integral for transition probability density

$$
K\left(x, x_{0}, t, t_{0}\right)=\int_{x_{0}}^{x} \mathcal{D} x(\tau) \exp \left\{-\frac{1}{2} \int_{t_{0}}^{t}\left(\dot{x}(\tau)-A_{\mathrm{ef}}(x(\tau))\right)^{2} d \tau-\frac{1}{2} \int_{t_{0}}^{t} A_{\mathrm{ef}}^{\prime}(x(\tau)) d \tau\right\} .
$$

$A_{\mathrm{ef}}^{\prime}(x(\tau))$ denotes derivative by argument and

$$
\mathcal{D} x(\tau)=\prod_{\tau} \frac{d x(\tau)}{\sqrt{2 \pi d \tau}} .
$$


Given transition probability density $K\left(x, x_{0}, t, t_{0}\right)$ let's write the following

$$
P(x, t)=\int K\left(x, x_{0}, t, t_{0}\right) P_{0}\left(x_{0}, t_{0}\right) d x_{0} .
$$

Here transition probabilities density $P(x, t)$ and $P_{0}\left(x_{0}, t_{0}\right)$ are given at respective time moments and are normalized to 1 . To transform equation (1) to transition probability density one needs to perform a variable substitution in (14) using connection between variables (9)

$$
P(r, t)=\int \tilde{K}\left(r, r_{0}, t, t_{0}\right) P_{0}\left(r_{0}, t_{0}\right) d r_{0}
$$

where

$$
\tilde{K}\left(r, r_{0}, t, t_{0}\right)=\frac{1}{B(r)} K\left(\varphi(r), \varphi\left(r_{0}\right), t, t_{0}\right) .
$$

Formulas (15) and (12) as solution of the problem were presented in [7]. However, given formulas are not comfortable in application because in order to receive transition probability density for stochastic equation (1) one needs to perform additional transformation. That is why it is convenient to express transition probability density for stochastic process (1) with the help of path integral of variables $r(\tau)$. Let's perform variable substitution in path integral (12) according to $(9)(x(\tau)=\phi(r(\tau)))$. In particular the following is received for expressions in integral (12)

$$
\begin{aligned}
\dot{x}(\tau) & =\frac{1}{B(r(\tau))} \dot{r}(\tau), \quad A_{\mathrm{ef}}(x(\tau))=\frac{A(r(\tau))}{B(r(\tau))}-\frac{1}{2} B^{\prime}(r(\tau)), \\
A_{\mathrm{ef}}^{\prime}(x(\tau)) & =A^{\prime}(r(\tau))-A(r(\tau)) \frac{B^{\prime}(r(\tau))}{B(r(\tau))}-\frac{1}{2} B(r(\tau)) B^{\prime \prime}(r(\tau)) .
\end{aligned}
$$

A measure element (13) is the next

$$
\mathcal{D} r(\tau)=\prod_{\tau} \frac{d r(\tau)}{\sqrt{2 \pi B^{2}(r(\tau)) d \tau}}
$$

Substituting denoted transformations into (12) we receive expression for transition probability density of stochastic process $(1)$

$$
\tilde{K}\left(r, r_{0}, t, t_{0}\right)=\frac{1}{B(r)} \int_{r_{0}}^{r} \mathcal{D} r(\tau) \exp \left(-\frac{1}{2} \int_{t_{0}}^{t}\left(\frac{\dot{r}(\tau)-A(r(\tau))}{B(r(\tau))}+\frac{1}{2} B^{\prime}(r(\tau))\right)^{2} d \tau-\int_{t_{0}}^{t} u(r(\tau)) d \tau\right) .
$$

Here the following is denoted

$$
u(r(\tau))=\frac{1}{2} A^{\prime}(r(\tau))-\frac{1}{2} A(r(\tau)) \frac{B^{\prime}(r(\tau))}{B(r(\tau))}-\frac{1}{4} B(r(\tau)) B^{\prime \prime}(r(\tau)) .
$$

Let's extract the item $\frac{1}{2} B^{\prime}(r(\tau))$ in quadratic expression exponentially in (16). For item that contains multiplicator $\dot{r}(\tau)$, we receive the following

$$
\exp \left(-\frac{1}{2} \int_{t_{0}}^{t} \dot{r}(\tau) \frac{B^{\prime}(r(\tau))}{B(r(\tau))} d \tau\right)=\exp \left(-\frac{1}{2} \int_{t_{0}}^{t} \frac{d \ln (B(r(\tau)))}{d \tau} d \tau\right)=\sqrt{\frac{B\left(r_{0}\right)}{B(r)}}
$$

As a result for transition probability density we receive the following path integral

$$
\tilde{K}\left(r, r_{0}, t, t_{0}\right)=\sqrt{\frac{B\left(r_{0}\right)}{B(r)^{3}}} \int_{r_{0}}^{r} \mathcal{D} r(\tau) \exp \left(-\frac{1}{2} \int_{t_{0}}^{t}\left(\frac{\dot{r}(\tau)-A(r(\tau))}{B(r(\tau))}\right)^{2} d \tau-\int_{t_{0}}^{t} u_{0}(r(\tau)) d \tau\right)
$$

with the following notations

$$
u_{0}(r(\tau))=\frac{1}{2} A^{\prime}(r(\tau))-A(r(\tau)) \frac{B^{\prime}(r(\tau))}{B(r(\tau))}+\frac{1}{8} B^{\prime}(r(\tau))^{2}-\frac{1}{4} B(r(\tau)) B^{\prime \prime}(r(\tau)) .
$$


It can be seen that for constant volatility $B(r(\tau))=$ const in expression for (18) only the first item remains which corresponds to (32).

Accordingly, we receive an integral path representation of transition probability density corresponding to stochastic equation (1). The received formula is convenient in application because it allows receiving a path integral by direct substitution of respective values of the stochastic equation. Based on (17) one can receive transition probabilities density for stochastic processes which are used in financial engineering. Examples for Brownian geometric motion and Cox-Ingersoll-Ross (CIR) process [16] are given in Appendix B.

As we already noted, the Ito rule was used to obtaine formulas (17) for transition probability density. As it is known [2] the Fokker-Plank equation for transition probability density corresponding to stochastic equation (1) is

$$
\frac{\partial K\left(r, r_{0}, t, t_{0}\right)}{\partial t}=-\mathcal{H} K\left(r, r_{0}, t, t_{0}\right)
$$

where

$$
\mathcal{H}=-\frac{1}{2} \frac{\partial^{2}}{\partial r^{2}} B(r)^{2}+\frac{\partial}{\partial r} A(r)
$$

is a differential operator for Fokker-Plank equation. It is understood that path integrals for transition probability density which are received base on stochastic equation and Fokker-Plank equation must match.

The path integral for transition probability density that was found based on the solution of the Fokker-Plank equation is given in Appendix C. As it can be seen from solution (50), (51) function $u_{0}(r(\tau))$ matches the one for path integral (51) which means that both approaches give the same results.

Let's point out that in work [10] the path integral for Fokker-Plank equation (19) was defined based on quantum-mechanical analogies and Feynman's integral in phase space (coordinates and impulses space) for imaginary time. Herein a certain "symmetry" rule was used for an operator in FokkerPlank equation (20). A function of coordinates and impulses and of Feynman's integral in phase space conforms to symmetry operator. After calculating path integrals for impulses, an action (expression in the exponent of formula (50)) and corresponding Lagrange function (which in physical researches is known by the name of Onsager-Machlup function) was received. However, the received expression differs vastly from the one in (50). The symmetry approach using the Weyl rule was used in [11] where the Fokker-Plank equation for particle dynamics under few noncorrelated Brownian motions was considered. If to limit to a single coordinate of the particle and to one Brownian motion, we will receive equation of the form (19) with the following operator

$$
\mathcal{H}=-\frac{1}{2}\left(\frac{\partial}{\partial r} B(r)\right)^{2}+\frac{\partial}{\partial r} A(r)
$$

Operator (21) can be reduced to the form (20)

$$
\mathcal{H}=-\frac{1}{2} \frac{\partial^{2}}{\partial r^{2}} B(r)^{2}+\frac{\partial}{\partial r} \tilde{A}(r)
$$

with notation $\tilde{A}(r)=A(r)+\frac{1}{2} B(r) B^{\prime}(r)$.

For Fokker-Plank equation with operator (22) based on (50) we receive following solution

$$
\begin{aligned}
K\left(r, r_{0}, t, t_{0}\right)= & \sqrt{\frac{B\left(r_{0}\right)}{B(r)}} \int \mathcal{D} \nu(\tau) \exp \left\{-\frac{1}{2} \int_{t_{0}}^{t}\left(\frac{\nu(\tau)-A(r(\tau))}{B(r(\tau))}\right)^{2} d \tau-\int_{t_{0}}^{t} \bar{u}_{0}(r(\tau)) d \tau\right\} \\
& \times \delta\left(r-r_{0}-\int_{t_{0}}^{t} \nu(\tau) d \tau\right), \\
\bar{u}_{0}(r(\tau))= & \frac{1}{2} A^{\prime}(r(\tau))-\frac{1}{2} A(r(\tau)) \frac{B^{\prime}(r(\tau))}{B(r(\tau))} .
\end{aligned}
$$


Comparing expression (24) with respective results of the work [11] we can see that the (24) has missing term $\frac{1}{8} B^{\prime}(r(\tau))^{2}$. It is obvious that the difference in works $[10,11]$ is caused by the arbitrariness in symmetrization procedures used. At the same time, our approach of construction of a solution to the Fokker-Plank equation is based only on identical transformation. Let us notice that in work [13] in case of Stratonovich scheme an action with Onsager-Machlup function was received which matches (23), (24).

Let's generalize the considered approach of construction of path integral for a stochastic process defined by two or more equations. In particular, let's consider a system of two stochastic equations

$$
d r_{i}(\tau)=A_{i}\left(r_{i}(\tau)\right) d \tau+B_{i}\left(r_{i}(\tau)\right) d w_{i}(\tau), \quad i=1,2
$$

If Wiener's processes $w_{i}(i=1,2)$ are independent of each other $\left\langle d w_{i}\right\rangle=0,\left\langle d w_{i}^{2}\right\rangle=d t(i=1,2)$, $\left\langle d w_{1} d w_{2}\right\rangle=0$ then we receive a multiplication of transition probabilities density for each stochastic equation which are defined by formulas (16), (18) respectively including parameters.

In case of correlation of Wiener's processes $\left\langle d w_{1} d w_{2}\right\rangle=\rho$ for means one can receive a form with double path integral

$$
\int_{\mathbf{w}_{0}} d \mu(\mathbf{w}) F(\mathbf{r}(\tau))=\int_{\mathbf{r}_{0}} d \mu(\mathbf{r}) F(\mathbf{r}(\tau))
$$

Elements of Wiener's measure for two correlated Wiener's processes [3] are denoted as

$$
\begin{aligned}
d \mu(\mathbf{w}) & =\mathcal{D} \mathbf{w}(\tau) \exp \left(-\frac{1}{2} \int_{t_{0}}^{t} \dot{\mathbf{w}}(\tau) \operatorname{Cov}^{-1} \dot{\mathbf{w}}(\tau) d \tau\right), \\
\mathcal{D} \mathbf{w}(\tau) & =\prod_{\tau} \frac{d w_{1}(\tau)}{\sqrt{2 \pi d \tau}} \frac{d w_{2}(\tau)}{\sqrt{2 \pi d \tau}}(\operatorname{det} \operatorname{Cov})^{-1},
\end{aligned}
$$

where

$$
\operatorname{Cov}=\left(\begin{array}{ll}
1 & \rho \\
\rho & 1
\end{array}\right)
$$

is a covariant matrix of Wiener's processes and also $\dot{\mathbf{w}}(\tau)=\left(\dot{w}_{1}(\tau), \dot{w}_{2}(\tau)\right)$. Using the above approach one can receive a measure in a space of variables $r_{1}(\tau), r_{2}(\tau)$ and a functional representation of transition probability density for variables given by equations $(25)$.

\section{Conclusions}

The work considers an application of the path integral method to determine transition probability density of stochastic variables and means values that occur in problems of financial engineering. A functional representation of transition probability density of stochastic variables, which is defined by a stochastic equation with drift and volatility as functions of a stochastic variable, was built. Path integrals are constructed by two different approaches. The first approach is based on stochastic equation and the other one is based on Fokker-Plank equation for the transition probability density of stochastic variable.

Construction of path integral based stochastic equation is based on variable subsection in Wiener's path integral (Wiener's measure). As in work [7] a transition is performed from the stochastic equation with random volatility to a stochastic equation of constant volatility using a variable substitution approach. This results in an unambiguously defined path integral. A form of path integral for stochastic equation depends on rule choice of stochastic integral calculating. We use the Ito rule, which is conventional in problems of financial engineering. Unlike work [7] we perform an inverse variable substitution in a path integral and receive a path integral in a variable of the initial stochastic equation.

In the second approach, the path integral for transition probability density is constructed on a solution of the Fokker-Plank equation which also conforms to the Ito rule. So we used operational 
approaches and Gauss path integral. It is shown that path integrals received by two different approaches are equivalent. Let's note that used approach doesn't have flaws inherent to a number of works where certain rules of symmetrization of Fokker-Plank differential operator were used, that led to inconsistent results. Application of received path integrals was shown on an example of financial problems like Black-Scholes formula for option and time structure of interest rate in CIR model. Methods considered in work can be generalized for problems with several stochastic equations, as a subject to separate research.

\section{Appendix A}

Let's rewrite equation (7) in integral form

$$
w(\tau)-w\left(t_{0}\right)=\frac{1}{\sigma}\left(r(\tau)-r\left(t_{0}\right)\right)-\frac{1}{\sigma} \int_{t_{0}}^{\tau} A\left(r\left(\tau^{\prime}\right)\right) d \tau^{\prime} .
$$

Based on relation between variables $w(\tau)$ and $r(\tau)(7)$ let's find a Jacobian for the variable substitution in path integral over Wiener's measure (formulas (4), (5), (6)). For the measure element we find that (3)

$$
\mathcal{D} w(\tau)=\tilde{\mathcal{D}} r(\tau)\left\|\frac{\delta w(\tau)}{\delta r\left(\tau^{\prime}\right)}\right\|, \quad \tilde{\mathcal{D}} r(\tau)=\prod_{\tau} \frac{d r(\tau)}{\sqrt{2 \pi d \tau}}
$$

where

$$
\left\|\frac{\delta w(\tau)}{\delta r\left(\tau^{\prime}\right)}\right\|=\left\|\frac{1}{\sigma} \delta\left(\tau-\tau^{\prime}\right)-\frac{1}{\sigma} A^{\prime}\left(r\left(\tau^{\prime}\right)\right) \theta\left(\tau-\tau^{\prime}\right)\right\|=\left(\prod_{\tau} \frac{1}{\sigma}\right) J_{0}
$$

is a functional determinant. The following notation was used

$$
J_{0}=\left\|\delta\left(\tau-\tau^{\prime}\right)-A^{\prime}\left(r\left(\tau^{\prime}\right)\right) \theta\left(\tau-\tau^{\prime}\right)\right\|,
$$

where the prime sign in $A^{\prime}(r)$ points to the derivative over argument. We consolidate the first multiplicator in (27) with element of the measure $\tilde{\mathcal{D}} r(\tau)$ in $(26)$ and write the following

$$
\mathcal{D} r(\tau)=\tilde{\mathcal{D}} r(\tau)\left(\prod_{\tau} \frac{1}{\sigma}\right)=\prod_{\tau} \frac{d r(\tau)}{\sqrt{2 \pi \sigma^{2} d \tau}} .
$$

The functional determinant (28) is obtained using identity $[11,19]$

$$
\operatorname{det}(I+K)=e^{\operatorname{Sp} \ln (I+K)}
$$

where $I$ and $K$ are unitary and arbitrary matrices in functional space respectively and Sp denotes the spur of matrix. From formula (28)

$$
K\left(\tau-\tau^{\prime}\right)=-A^{\prime}\left(r\left(\tau^{\prime}\right)\right) \theta\left(\tau-\tau^{\prime}\right) .
$$

Application of formula (29) consists in expansion of $\ln (I+K)$ into series and in finding of spur of powers of matrices $K\left(\tau-\tau^{\prime}\right)$. In presence of multiplicator $\theta\left(\tau-\tau^{\prime}\right)$ in (30) we receive the following for all matrix powers starting from second

$$
\operatorname{Sp} K^{n}=0, \quad n \geqslant 2
$$

As a result we find the following for the determinant (28)

$$
J_{0}=\exp \left\{\left.\int_{t_{0}}^{t} K\left(\tau-\tau^{\prime}\right)\right|_{\tau^{\prime} \rightarrow \tau} d \tau\right\}=\exp \left\{-\frac{1}{2} \int_{t_{0}}^{t} A^{\prime}(r(\tau)) d \tau\right\} .
$$

Mathematical Modeling and Computing, Vol. 9, No. 1, pp. 166-177 (2022) 
It was taken into account in formula (31) that $\left.\theta\left(\tau-\tau^{\prime}\right)\right|_{\tau^{\prime} \rightarrow \tau}=\frac{1}{2}$ while keeping in mind that $\left.\theta\left(\tau-\tau^{\prime}\right)\right|_{\tau^{\prime} \rightarrow \tau}=\int_{t_{0}}^{\tau} \delta\left(\tau-\tau_{1}\right) d \tau_{1}=\frac{1}{2}$.

As a result, we receive the following path integral for the transition probability density of a stochastic process

$$
K\left(r, r_{0}, t, t_{0}\right)=\int \mathcal{D} r(\tau) \exp \left\{-\frac{1}{2 \sigma^{2}} \int_{t_{0}}^{t}(\dot{r}(\tau)-A(r(\tau)))^{2} d \tau-\frac{1}{2} \int_{t_{0}}^{t} A^{\prime}(r(\tau)) d \tau\right\} .
$$

Formula (32) was also obtained in [7] where for the variable substitution Jacobian the method of finite approximations was used.

\section{Appendix B}

\section{B.1. Geometric Brownian motion}

Let's consider stochastic equation for geometric brownian motion $[2,16]$

$$
d S(\tau)=\phi S(\tau) d \tau+\sigma S(\tau) d w(\tau)
$$

where stochastic variable $S(\tau) \geqslant 0, \tau \in\left[t_{0}, t\right], \sigma \geqslant 0$. Substituting values $A(S(\tau))=\phi S(\tau), B(S(\tau))=$ $\sigma S(\tau)$ of stochastic equation (33) into formula (16) we receive the following path integral for transition probability density

$$
K_{\mathrm{GB}}\left(S, S_{0}, t, t_{0}\right)=\frac{1}{\sigma S} \sqrt{\frac{S_{0}}{S}} \exp \left(\frac{1}{2}\left(t-t_{0}\right)\left(\phi-\frac{1}{4} \sigma^{2}\right)\right) \int_{S_{0}}^{S} \mathcal{D} S(\tau) \exp \left(-\frac{1}{2} \int_{t_{0}}^{t}\left(\frac{\dot{S}-\phi S(\tau)}{\sigma S(\tau)}\right)^{2} d \tau\right) .
$$

Path integral (34) is reduced to a known integral $[4,21]$ by variable substitution $x(\tau)=\ln (S(\tau))$. As a result we receive the following

$$
K_{G B}\left(S, S_{0}, t, t_{0}\right)=\frac{1}{\sqrt{2 \pi\left(t-t_{0}\right)} \sigma S} \sqrt{\frac{S_{0}}{S}} \exp \left(\frac{1}{2}\left(t-t_{0}\right)\left(\phi-\frac{1}{4} \sigma^{2}\right)\right) \exp \left(-\frac{1}{2} \frac{\left(\ln \left(\frac{S}{S_{0}}\right)-\phi\left(t-t_{0}\right)\right)^{2}}{\sigma^{2}\left(t-t_{0}\right)}\right) .
$$

As it is known [16] that geometric brownian motion is used in determination of option pricing (see (4))

$$
C\left(S_{0}\right)=\int_{0}^{\infty} K_{G B}\left(S, S_{0}, t, t_{0}\right) F(S) d S,
$$

where $F(S)$ is a payoff function.

Substituting $F(S)=(S-K)^{+}(K$ is strike price, sign + denotes that $F(S)>0$ when $S>K$ and equals to zero otherwise), we receive a well-known Black-Scholes formula [16] for European option price.

\section{B.2. Stochastic equation CIR}

The CIR model is represented by equation

$$
d r(\tau)=k(\theta-r(\tau)) d \tau+\sigma \sqrt{r(\tau)} d w(\tau)
$$

where stochastic variable is $r(\tau) \geqslant 0, \tau \in\left[t_{0}, t\right], \sigma \geqslant 0, k \geqslant 0, \theta \geqslant 0$. Using (16) it is received an expression for transition probability density

$$
K_{\mathrm{CIR}}\left(r, r_{0}, t, t_{0}\right)=\frac{\left(\frac{r}{r_{0}}\right)^{\frac{\lambda}{2}}}{\sigma\left(r r_{0}\right)^{\frac{1}{4}}} \exp \left\{-\frac{k}{\sigma^{2}}\left(r-r_{0}\right)\right\} \exp \left\{\frac{k^{2} \theta}{\sigma^{2}}\left(t-t_{0}\right)\right\}
$$




$$
\times \int_{r_{0}}^{r} \mathcal{D} r(\tau) \exp \left\{-\frac{1}{2 \sigma^{2}} \int_{t_{0}}^{t} \frac{\dot{r}(\tau)^{2}}{r(\tau)} d \tau-\frac{k^{2}}{2 \sigma^{2}} \int_{t_{0}}^{t} r(\tau) d \tau-\frac{\sigma^{2}\left(\lambda^{2}-\frac{1}{4}\right)}{8} \int_{t_{0}}^{t} \frac{1}{r(\tau)} d \tau\right\},
$$

where $\lambda=\frac{2 k \theta}{\sigma^{2}}-1$. Let's consider the following path integral found in (35)

$$
I\left(r, r_{0}\right)=\int_{r_{0}}^{r} \mathcal{D} r(\tau) \exp \left\{-\frac{1}{2 \sigma^{2}} \int_{t_{0}}^{t} \frac{\dot{r}(\tau)^{2}}{r(\tau)} d \tau-\frac{k^{2}}{2 \sigma^{2}} \int_{t_{0}}^{t} r(\tau) d \tau-\frac{\sigma^{2}\left(\lambda^{2}-\frac{1}{4}\right)}{8} \int_{t_{0}}^{t} \frac{1}{r(\tau)} d \tau\right\} .
$$

The path integral (36) is reduced to radial oscillatory integral $[4,21]$ using variable substitution $r(\tau)=$ $\frac{\sigma^{2}}{4} z(\tau)^{2}$

$$
I\left(r, r_{0}\right)=\int_{\frac{2 \sqrt{r_{0}}}{\sigma}}^{\frac{2 \sqrt{r}}{\sigma}} \mathcal{D} z(\tau) \exp \left\{-\frac{1}{2} \int_{t_{0}}^{t} \dot{z}(\tau)^{2} d \tau-\frac{\omega^{2}}{2} \int_{t_{0}}^{t} z(\tau)^{2} d \tau-\frac{\left(\lambda^{2}-\frac{1}{4}\right)}{2} \int_{t_{0}}^{t} \frac{1}{z(\tau)^{2}} d \tau\right\} .
$$

Notation $\omega=\frac{k}{2}$ is introduced in (37). For the integral above, we find the following

$$
I\left(r, r_{0}\right)=\frac{2 \omega}{\sigma \sinh \left(\omega\left(t-t_{0}\right)\right)}\left(r r_{0}\right)^{\frac{1}{4}} \exp \left\{-\frac{2 \omega}{\sigma^{2}}\left(r+r_{0}\right) \operatorname{coth}\left(\omega\left(t-t_{0}\right)\right)\right\} I_{\lambda}\left(\frac{4 \omega \sqrt{r r_{0}}}{\sigma^{2} \sinh \left(\omega\left(t-t_{0}\right)\right)}\right)
$$

where $I_{\lambda}(x)$ is Bessel function. In summary, for the CIR model of transition probability density, the following expression is obtained

$$
\begin{aligned}
K_{\mathrm{CIR}}\left(r, r_{0}, t, t_{0}\right)= & \frac{k \exp \left\{\frac{k^{2} \theta}{\sigma^{2}}\left(t-t_{0}\right)\right\}}{\sigma^{2} \sinh \left(\frac{1}{2} k\left(t-t_{0}\right)\right)}\left(\frac{r}{r_{0}}\right)^{\frac{\lambda}{2}} \exp \left\{-\frac{k}{\sigma^{2}}\left(\left(r-r_{0}\right)+\left(r+r_{0}\right) \operatorname{coth} \frac{k\left(t-t_{0}\right)}{2}\right)\right\} \\
& \times I_{\lambda}\left(\frac{2 k \sqrt{r r_{0}}}{\sigma^{2} \sinh \left(\frac{1}{2} k\left(t-t_{0}\right)\right)}\right)
\end{aligned}
$$

It is easy to see that (39) matches the [16] which was found using a different approach.

In models of interest rate path integrals with $F(x)=e^{-x}$ occur (see also formula (6)). Then in path integral (37) one should take into account a multiplier $\exp \left\{-\int_{t_{0}}^{t} r(\tau) d \tau\right\}$. It is easy to see that mentioned path integral is reduced to (38) with substitution $\omega=\frac{\sqrt{k^{2}+2 \sigma^{2}}}{2}$. Taking that into account and integrating final expression for $r \in[0, \infty]$ we receive a structure of interest rate in CIR model [16].

\section{Appendix C}

\section{Path integral for Fokker-Plank equation}

Let's set solution to the (19) equation in the form of exponent operator

$$
K\left(r, r_{0}, t, t_{0}\right)=e^{-\left(t-t_{0}\right) \mathcal{H}} \delta\left(r-r_{0}\right) .
$$

Transform differential operator (20) with the identity approach

$$
\mathcal{H}=-\frac{1}{2}\left(B(r) \frac{\partial}{\partial r}-p(r)\right)^{2}+u(r)
$$

where

$$
\begin{aligned}
& p(r)=\frac{A(r)}{B(r)}-\frac{3}{2} B^{\prime}(r), \\
& u(r)=\frac{1}{2} A^{\prime}(r)-A(r) \frac{B^{\prime}(r)}{B(r)}+\frac{1}{2} \frac{A(r)^{2}}{B(r)^{2}}+\frac{1}{8} B^{\prime}(r)^{2}-\frac{1}{4} B(r) B^{\prime \prime}(r) .
\end{aligned}
$$


Using Gaussian quadrature $[19,20]$ for linearization of quadratic term of operator (41) in formula (40) results as

$$
\begin{aligned}
K\left(r, r_{0}, t, t_{0}\right)= & \int \mathcal{D} q(\tau) \exp \left\{-\frac{1}{2} \int_{t_{0}}^{t} q(\tau)^{2} d \tau\right\} \mathrm{T} \exp \left\{-\int_{t_{0}}^{t} q(\tau)\left(B(r) \frac{\partial}{\partial r}-p(r)\right) d \tau-\int_{t_{0}}^{t} u(r) d \tau\right\} \\
& \times \delta\left(r-r_{0}\right) .
\end{aligned}
$$

Symbol T denotes a T-product (a chronological ordering of operators) $[4,18]$ and also the following notation was used

$$
\mathcal{D} q(\tau)=\prod_{\tau} \sqrt{\frac{d \tau}{2 \pi}} d q(\tau) .
$$

After "unraveling" the differential operator $B(r) \frac{\partial}{\partial r}[18-20]$ in (43) one can get

$$
\begin{aligned}
K\left(r, r_{0}, t, t_{0}\right)= & \int \mathcal{D} q(\tau) \exp \left\{-\frac{1}{2} \int_{t_{0}}^{t} q(\tau)^{2} d \tau\right\} \exp \left\{\int_{t_{0}}^{t} q(\tau) p(r(\tau)) d \tau-\int_{t_{0}}^{t} u(r(\tau)) d \tau\right\} \\
& \times \delta\left(r\left(t_{0}\right)-r_{0}\right) .
\end{aligned}
$$

In (44) the following was denoted

$$
r(\tau)=\exp \left\{-\int_{\tau}^{t} q\left(\tau^{\prime}\right) d \tau^{\prime} B(r) \frac{\partial}{\partial r}\right\} r \exp \left\{\int_{\tau}^{t} q\left(\tau^{\prime}\right) d \tau^{\prime} B(r) \frac{\partial}{\partial r}\right\} .
$$

It is easy to see that $r(\tau)$ satisfies equation

$$
\dot{r}(\tau)=q(\tau) B(r(\tau)), \quad r(t)=r .
$$

Solution of the equation (45) is the following

$$
r(\tau)=f^{-1}\left(f(r)-\int_{\tau}^{t} q\left(\tau^{\prime}\right) d \tau^{\prime}\right)
$$

where function $f(r)$ is a solution to equation $f^{\prime}(r)=\frac{1}{B(r)}$ and $f^{-1}(r)$ denotes inverse function such that $f^{-1}(f(r))=r$.

In order to give a usual form to integral (44) let's perform a variable substitution

$$
f^{-1}\left(f(r)-\int_{\tau}^{t} q\left(\tau^{\prime}\right) d \tau^{\prime}\right)=r-\int_{\tau}^{t} \nu\left(\tau^{\prime}\right) d \tau^{\prime}
$$

Differentiating (46) for $\tau$ we receive the following relation between variables

$$
q(\tau)=\frac{\nu(\tau)}{B\left(r-\int_{\tau}^{t} \nu\left(\tau^{\prime}\right) d \tau^{\prime}\right)} .
$$

Similarly to Appendix A Jacobian is defined for variable substitution in path integral (44)

$$
\left\|\frac{\delta q(\tau)}{\delta \nu\left(\tau^{\prime}\right)}\right\|=\left\|\frac{\delta\left(\tau-\tau^{\prime}\right)}{B(r(\tau))}+\nu(\tau) \frac{B^{\prime}(r(\tau))}{B(r(\tau))^{2}} \theta\left(\tau-\tau^{\prime}\right)\right\|=\prod_{\tau} \frac{1}{B(r(\tau))} J_{0} .
$$

In formula (47) the following is denoted

$$
J_{0}=\left\|\delta\left(\tau-\tau^{\prime}\right)+\nu(\tau) \frac{B^{\prime}(r(\tau))}{B(r(\tau))} \theta\left(\tau^{\prime}-\tau\right)\right\|,
$$

where $\theta\left(\tau-\tau^{\prime}\right)$ is a Heaviside function. Using calculation approach shown in appendix A for $J_{0}$ we receive

$$
J_{0}=\exp \left(\left.\int_{t_{0}}^{t} \nu(\tau) \frac{B^{\prime}(r(\tau))}{B(r(\tau))} \theta\left(\tau^{\prime}-\tau\right)\right|_{\tau^{\prime} \rightarrow \tau} d \tau\right)=\sqrt{\frac{B(r)}{B\left(r_{0}\right)}} .
$$


As a result for transition probability density

$$
\begin{aligned}
K\left(r, r_{0}, t, t_{0}\right)= & \sqrt{\frac{B(r)}{B\left(r_{0}\right)}} \int \mathcal{D} \nu(\tau) \exp \left\{-\frac{1}{2} \int_{t_{0}}^{t} \frac{\nu(\tau)^{2}}{B(r(\tau))^{2}} d \tau\right\} \\
& \times \exp \left\{\int_{t_{0}}^{t} \nu(\tau) \frac{p(r(\tau))}{B(r(\tau))} d \tau-\int_{t_{0}}^{t} u(r(\tau)) d \tau\right\} \delta\left(r-r_{0}-\int_{t_{0}}^{t} \nu(\tau) d \tau\right) .
\end{aligned}
$$

In formulas above the following was denoted

$$
r(\tau)=r-\int_{\tau}^{t} \nu\left(\tau^{\prime}\right) d \tau^{\prime}, \quad \mathcal{D} \nu(\tau)=\prod_{\tau} \sqrt{\frac{d \tau}{2 \pi}} \frac{d \nu(\tau)}{B(r(\tau))}
$$

Let's perform some transformation in exponent expression of (48) formula in term containing $p(r(\tau))$. Combine the first term in $p(r(\tau))$ (42) with multiplicator $\nu(\tau)$ with quadratic term $\nu(\tau)^{2}$ in formula (48). The second term in $p(r)$ we calculate in closed form

$$
\exp \left(-\frac{3}{2} \int_{t_{0}}^{t} \nu(\tau) \frac{B^{\prime}(r(\tau))}{B(r(\tau))} d \tau\right)=\exp \left(-\frac{3}{2} \int_{t_{0}}^{t} \frac{d}{d \tau} \ln (B(r(\tau))) d \tau\right)=\left(\frac{B\left(r_{0}\right)}{B(r)}\right)^{\frac{3}{2}}
$$

As a result of the above transformation we receive the following path integral for transition probability density

$$
\begin{aligned}
K\left(r, r_{0}, t, t_{0}\right)= & \frac{B\left(r_{0}\right)}{B(r)} \int \mathcal{D} \nu(\tau) \exp \left\{-\frac{1}{2} \int_{t_{0}}^{t}\left(\frac{\nu(\tau)-A(r(\tau))}{B(r(\tau))}\right)^{2} d \tau-\int_{t_{0}}^{t} u_{0}(r(\tau)) d \tau\right\} \\
& \times \delta\left(r-r_{0}-\int_{t_{0}}^{t} \nu(\tau) d \tau\right) .
\end{aligned}
$$

Value $u_{0}(r(\tau))$ comparing to $u(r(\tau))(42)$ does not include term with $A(r(\tau))^{2}$

$$
u_{0}(r(\tau))=\frac{1}{2} A^{\prime}(r(\tau))-A(r(\tau)) \frac{B^{\prime}(r(\tau))}{B(r(\tau))}+\frac{1}{8} B^{\prime}(r(\tau))^{2}-\frac{1}{4} B(r(\tau)) B^{\prime \prime}(r(\tau)) .
$$

Path integral (50) is defined in space of "velocities" $\nu(\tau)[4,8,17,19]$ where connection with "coordinate" $r(\tau)$ is defined in (49). Path integrals (16) and (50) give us equivalent solutions of the problem. We use the form of path integral (50) only for comparison of approaches based on stochastic equation (1) and a respective Fokker-Plank equation (19).

[1] Gardiner C. W. Handbook of Stochastic Methods for Physics, Chemistry and the Natural Sciences. Springer Series in Synergetics (2004).

[2] Øksendal B. K. Stochastic differential equations: an introduction with applications. Springer-Verlag, Berlin, Heidelberg (2003).

[3] Chaichian M., Demichev A. Path integrals in physics. Stochastic processes and quantum mechanics. Taylor \& Francis (2001).

[4] Kleinert H. Path integrals in quantum mechanics, statistics, polymer physics and financial markets. World Scientific Publishing Co. (2004).

[5] Chaichian M., Demichev A. Path integrals in physics. QFT, statistical physics and modern applications. Taylor \& Francis (2001).

[6] Linetsky V. The Path Integral Approach to Financial Modeling and Options Pricing. Computational Economics. 11, 129-163 (1998).

[7] Goovaerts M., De Schepper A., Decamps M. Closed-form approximations for diffusion densities: a path integral approach. Journal of Computational and Applied Mathematics. 164-165, 337-364 (2004).

[8] Yanishevskyi V. S., Nodzhak L. S. The path integral method in interest rate models. Mathematical Modeling and Computing. 8 (1), 125-136 (2021). 
[9] Baaquie B. E. Quantum finance. Path Integrals and Hamiltonians for Options and Interest Rates. Cambridge University Press, New York (2004).

[10] Jurisch A. Lagrangian dynamics in inhomogeneous and thermal environments. An application of the Onsager-Machlup theory I. Preprint arXiv:2012.06820v1 (2020).

[11] Arnold P. Symmetric path integrals for stochastic equations with multiplicative noise. Physical Review E. 61, 6099 (2000).

[12] Bressloff P. C. Construction of stochastic hybrid path integrals using operator methods. Journal of Physics A: Mathematical and Theoretical. 54 (18), 185001 (2021).

[13] Cugliandolo L. F., Lecomte V., van Wijland F. Building a path-integral calculus: a covariant discretization approach. Journal of Physics A: Mathematical and Theoretical. 52, 50LT01 (2019).

[14] Cugliandolo L. F., Lecomte V. Rules of calculus in the path integral representation of white noise Langevin equations: the Onsager-Machlup approach. Journal of Physics A: Mathematical and Theoretical. 50, 345001 (2017).

[15] Arenas Z. G., Barci D. G. Functional integral approach for multiplicative stochastic processes. Physical Review E. 81, 051113 (2010).

[16] Lyuu Y.-D. Financial Engineering and Computation: Principles, Mathematics, and Algorithms. Cambridge University Press (2004).

[17] Yanishevsky V. S. Application of the path integral method to some stochastic models of financial engineering. Journal of Physical Studies. 25 (2), 2801 (2021).

[18] Nazajkinsky B. J., Sternin B. Y., Shalatov B. J. The methods of non-commutive analysis. Moscov, Tehnosfera (2002), (in Russian).

[19] Blazhievskii L. F. Path integrals and ordering of operators. Theoretical and Mathematical Physics. 40, 596-604 (1979).

[20] Blazhyevskyi L. F., Yanishevsky V. S. The path integral representation kernel of evolution operator in Merton-Garman model. Condensed Matter Physics. 14 (2), 23001 (2011).

[21] Grosche C., Steiner F. Handbook of Feynman Path Integrals. Springer, Berlin, Heidelberg (1998).

\title{
Метод функціонального інтегрування в стохастичних рівняннях фінансової інженерії
}

\author{
Янішевський В. С., Барановська С. П. \\ Начіональний університет "Львівська політехніка", \\ вул. С. Бандери, 12, 79013, Львів, Україна
}

\begin{abstract}
Метод функціонального інтегрування застосовано для визначення деяких середніх випадкових величини, що зустрічаються в задачах фінансової інженерії. Випадкова величина задається стохастичним рівнянням, де дрейф та волатильність $є$ функціями випадкової величини. В результаті для густини умовної ймовірності побудовано функціональний інтеграл шляхом заміни змінних у функціональному інтегралі Вінера (мірі Вінера). Для стохастичного рівняння використано правило Іто для інтерпретації стохастичного інтегралу. Функціональний інтеграл для густини умовної ймовірності знайдено також у результаті розв'язку рівняння Фоккера-Планка, що відповідає стохастичному рівнянню. Показано, що два підходи дають еквівалентні результати.
\end{abstract}

Ключові слова: стохастичне рівняння, густина умовної ймовірності, рівняння Фоккера-Планка, функціональний інтеграл. 